Published in final edited form as:

Arch Womens Ment Health. 2018 August ; 21(4): 411-419. doi:10.1007/s00737-018-0809-2.

\title{
Longitudinal trajectories of antidepressant use in pregnancy and the postnatal period
}

\author{
Gretchen Bandoli ${ }^{1}$, Grace M. Kuo ${ }^{2}$, Renu Sugathan ${ }^{3}$, Christina D. Chambers ${ }^{4}$, Matthieu \\ Rolland $^{5}$, and Kristin Palmsten ${ }^{1}$ \\ ${ }^{1}$ Department of Pediatrics, 9500 Gilman Drive MC 0828, University of California, San Diego, \\ 92093
}

2Skaggs School of Pharmacy and Pharmaceutical Sciences and Department of Family Medicine and Public Health, 9500 Gilman Drive, University of California, San Diego 92093

${ }^{3}$ Decision Support, University of California San Diego Health and Skaggs School of Pharmacy and Pharmaceutical Sciences and Department of Family Medicine and Public Health, 9500 Gilman Drive, University of California, San Diego 92093

${ }^{4}$ Department of Pediatrics and Family Medicine and Public Health, 9500 Gilman Drive MC 0828, University of California, San Diego, 92093

5University of Bordeaux, Inserm, Bordeaux Population Health Research Center, team IDLIC, UMR 1219, F-33000 Bordeaux, France

\begin{abstract}
Purpose-Studies of antidepressant safety in pregnancy typically do not address complex patterns of use throughout pregnancy. We performed longitudinal trajectory modeling to describe patterns of antidepressant use in the first 32 weeks of pregnancy, and test whether these trajectories are associated with a reduction in birth weight or gestational age at delivery.
\end{abstract}

Methods-Our study included 166 pregnant women with deliveries between 2011-2015 who were prescribed an antidepressant between 91 days prior to last menstrual period and 32 weeks of gestation. From electronic medical records, we estimated average daily dose and cumulative dose per week for the first 32 weeks of gestation and for the first 13 weeks postnatal. We clustered women with similar utilization patterns using k-means longitudinal modeling and assessed the associations between trajectory group and birth weight and gestational age at delivery.

Corresponding author Gretchen Bandoli, 9500 Gilman Drive, MC 0828, Department of Pediatrics, University of California, San Diego, La Jolla, CA 92093, gbandoli@ucsd.edu.

Conflict of interest: Gretchen Bandoli declares that she has no conflicts of interest. Grace Kuo declares that she has no conflicts of interest. Renu Sugathan declares that she has no conflicts of interest. Christina Chambers declares that she has no conflicts of interest. Matt Rolland declares that he has no conflicts of interest. Kristin Palmsten declares that she has no conflicts of interest.

Compliance with Ethical Standards

The authors received no funding for this study.

Research involving human subjects: The article does not contain any studies with human participants or animals performed by any of the authors.

Informed consent: As this was retrospective data, informed consent was not required. 
Results-We identified four cumulative dose trajectory groups and three average daily dose trajectory groups in each period. Relative to the lowest trajectory group, the highest trajectory group during pregnancy was associated with reduced birth weight in multivariable analysis (average daily highest trajectory vs. lowest trajectory $\beta-314.1 \mathrm{~g}, 95 \% \mathrm{CI}-613.7,-15.5$ ) adjusted for depression severity score, maternal age, race, and pregnancy smoking. Trajectory groups were not associated with gestational age at delivery.

Conclusions-The highest trajectory group of antidepressant use in pregnancy was associated with a modest reduction in birth weight but not with gestational age at delivery. Longitudinal trajectories allow for a dynamic visualization and quantification of medication use among pregnant women.

\section{Keywords}

pharmacoepidemiology; pregnancy; antidepressive agents; birth outcomes; methodology

\section{Introduction}

Currently, between 6 and $9 \%$ of pregnant women use antidepressants at some point in pregnancy (Cooper et al. 2007; Andrade et al. 2008; Huybrechts et al. 2013). Often, studies of the reproductive safety of antidepressant use during pregnancy classify exposure dichotomously as any use in pregnancy, or any use in each of the three trimesters. Defining exposure in this manner is appealing because it reduces patterns of medication use into a few categories. However, the use of antidepressants in pregnancy is complex. Some women may choose non-pharmacologic approaches early in pregnancy because of concerns regarding cardiac birth defects. Further, although the prevalence of antidepressant use in pregnancy is increasing, the discontinuation of antidepressants due to pregnancy is also increasing (Ray and Stowe 2014). Reducing antidepressant exposure into dichotomous variables removes exposure information on changes in dose and agent, intensity of use, and coverage gaps that are important for understanding how antidepressants are used during pregnancy, and the associated pregnancy and birth outcomes.

Recent studies have used group trajectory methods to summarize complex individual level medication utilizations trajectories (Riegel et al. 2012; Franklin et al. 2013; Jacobs et al. 2015; Hurault-Delarue et al. 2016, 2017; Palmsten et al. 2017). Such trajectory methods can classify individuals with similar dose and frequency of use over time into groups. The strength of this approach is that it allows conversion of several correlated continuous variables into categorical variables that can then be used as predictors or outcomes in regression analyses (Genolini et al. 2015). To our knowledge, only three previous studies have assessed group trajectories of medication use during pregnancy (Hurault-Delarue et al. 2016, 2017; Palmsten et al. 2017). To date, the literature lacks information regarding patterns of gestational timing and dose specific to antidepressant use in pregnancy, whether these patterns are associated with pregnancy or birth outcomes, and whether patterns of antidepressant use in pregnancy predict postnatal antidepressant use.

We propose to identify patterns of antidepressant therapy across pregnancy using k-means longitudinal trajectory methods (Genolini et al. 2015). Using data abstracted from electronic 
medical records from University of California (UC) San Diego Health between 2011 and 2015 , the purpose of this paper is to use longitudinal trajectories to describe patterns of antidepressant prescriptions in pregnancy and the early postnatal period and to link prenatal antidepressant trajectories with perinatal outcomes. We hypothesized that cumulative dose trajectories with higher versus lower exposure levels would be associated with lower infant birth weight and shorter gestational age at delivery.

\section{Methods}

\section{Data source and study population}

Data for this study were abstracted from Epic, the electronic medical records software system used for individuals receiving care at UC San Diego Health. A trained Clinical Informatics Specialist performed a detailed search of inpatient labor and delivery flow chart and outpatient visit records. Data were abstracted and provided in a de-identified file to researchers. This study was approved by the Human Research Protections Program at UC San Diego.

Women with a delivery date on or before December 31, 2015 and a last menstrual period (LMP) date on or after April 1, 2011 were eligible for the study ( $n=9,331)$ (Figure 1). LMP was calculated from the gestational age at delivery in the labor and delivery flow chart (delivery date - gestational age at delivery). The sample was then limited to women who received at least one prescription for an antidepressant (specific medications listed in Online Resource 1) between LMP-91 days through the day before the delivery date ( $\mathrm{n}=186)$. Women who delivered before gestational week 20 were excluded $(n=2)$. To identify antidepressant use trajectories during the first 32 gestational weeks, we additionally excluded women with gestational age at delivery $<32$ weeks $(n=3)$ and women with an antidepressant prescription only after 32 gestational weeks $(n=15) .166$ pregnancies among 162 unique women were included in the study (5 twin deliveries, 4 sibling observations).

\section{Exposure measurement}

All antidepressant prescription dates were assigned to a pregnancy calendar with day 0 equal to 3 months before the LMP. Postnatal use was individualized from each woman's delivery date. We used antidepressant order dates and strength together with quantity prescribed and start dates, to estimate antidepressant dose on each gestational day. For each prescription, we compared 1) the number of days covered by the period between antidepressant order date and stop date with 2) the number of days covered by the prescription and allowable refills. The effective stop date was assigned as the shorter of the two periods. For any prescription which was noted as "patient reports no longer taking" we assigned one-half of the prescription coverage period. Prescriptions noted as "erroneous" or "patient refused" were assigned 0 days.

Dosages of all antidepressant agents were converted into fluoxetine dose equivalents (Hayasaka et al. 2015) and multiple agents per day were summed. Daily doses between LMP and delivery date were expressed as average daily dose per week, as were prescriptions 
between delivery date and 3 months after the delivery date. Finally, we created cumulative exposure per week from LMP-32 weeks and from delivery date-3 months postnatal.

\section{Trajectories}

In order to identify similar clusters of individual antidepressant trajectories, we employed the R statistical software package ' $\mathrm{km}$ ' (Genolini and Falissard 2016). Additional information on k-means longitudinal (kml) is found in Online Resource 1.

We applied the $\mathrm{kml}$ default settings, allowing k-means to run for 2 to 6 clusters 100 times each. Selection of the number of clusters was based upon the following factors: 1) optimization of the 3 quality criterion, 2) clinical relevance of the clusters, and 3) sample size in each cluster of at least 5 individuals. For pregnancy trajectories, we limited the exposure period to 32 weeks of gestation to avoid different exposure durations due to parturition. We performed k-means longitudinal on cumulative dose ( $\mathrm{mg} / \mathrm{day})$ per week from LMP up to 32 gestational weeks, and separately on average daily dose per week during the same period. There were 24 women with antidepressant prescriptions only in the 3 months prior to LMP, and we excluded them from the kml pregnancy trajectories, manually assigning them to a separate exposure group for the outcome analyses. Additionally, one outlier with a disproportionally high cumulative dose in the first 32 weeks of pregnancy $(16,974 \mathrm{mg})$ was removed from $\mathrm{kml}$ and manually coded into the highest trajectory group. For description of use in the postnatal period, we included all women with any antidepressant prescriptions between 3 months before the LMP and delivery, and performed k-means longitudinal on both the average cumulative dose per week in postnatal weeks 013 , and the average daily dose per week.

\section{Covariates}

A complete list of covariates abstracted from the electronic medical record is provided in Online Resource 1. Data on maternal characteristics, birth outcomes, Edinburgh Postnatal Depression Scale (EPDS) scores, and indication for antidepressant use were captured.

\section{Statistical analysis}

Descriptive characteristics (frequencies and means) were stratified by pregnancy cumulative exposure cluster $(n=5)$. We used chi-square with Fishers exact $p$-values to test whether trajectory group in pregnancy predicted trajectory group in the postnatal period. Also, to test the association between pregnancy trajectory group (average daily and cumulative) and maternal characteristics, we performed ANOVA with Tukey correction for multiple comparisons for continuous variables and Fishers exact tests for categorical variables.

We performed multivariable linear regression to estimate the associations of the cumulative and average daily dose pregnancy trajectory groups and birth weight and gestational weeks at birth. Based upon the small sizes of two of the cumulative trajectories, we collapsed them into one trajectory for multivariable regression. We only included EPDS scores administered between LMP and 32 weeks. We imputed 31 missing EPDS scores with the median EPDS observed value for each cumulative trajectory group. For the five multiple gestation pregnancies, we selected the infant with the lower birth weight. We considered race 
dichotomized as white vs. non-white, smoking in pregnancy, EPDS score, gestational day of EPDS administration, and maternal age at LMP as potential confounders. We created propensity scores by regressing the potential confounders on the trajectory categories. The probabilities of each trajectory were included as a linear continuous variable in the linear regression models. Robust standard errors were estimated to account for non-independence between sibling observations ( $\mathrm{n}=4$ women with two singleton pregnancies each). In a sensitivity analysis, we excluded twin pregnancies and repeated regression analyses.

Data cleaning and analysis were performed in SAS 9.4 (Carey, NC). Kml was performed in $\mathrm{R}$ version 3.4.0.

\section{Results}

The majority of women received a depression diagnosis before or during pregnancy $(80.1 \%)$, and $64.5 \%$ received a depression diagnosis between 3 months prior to LMP and 3 months after delivery. Approximately half (50.6\%) of the women received a diagnosis of anxiety in the same period. Few women had a diagnosis of pain disorder, and none received a diagnosis of a sleep disorder in the study period. Of 135 women with recorded EPDS scores in the first 32 weeks, $75 \%$ of them were administered in the first trimester. The median score was 6 with a range of $0-27$. (Table 1).

\section{Antidepressants and trajectories}

The most common class of antidepressant prescribed was selective serotonin reuptake inhibitors $(\mathrm{n}=78.3 \%)$, followed by bupropion $(13.9 \%)$, tricyclics, $(\mathrm{n}=8.4 \%)$, and other antidepressants $(7.8 \%$ ) (exceeds $100 \%$ due to some women taking more than one class of antidepressant).

\section{Pregnancy}

Of the 166 gestations, $142(85.5 \%)$ had at least one antidepressant prescription between LMP and week 32 of pregnancy. The remaining 24 gestations had at least one antidepressant prescription in the 3 months prior to LMP but did not have another between LMP and 32 weeks of gestation.

A. Cumulative pregnancy trajectory-Among gestations with an antidepressant prescription between LMP and 32 weeks, kml recommended 4 trajectory groups (Figure 2, top panel). Trajectory A ( $\mathrm{n}=87$ ) had a median cumulative fluoxetine equivalent dose of 534 $\mathrm{mg}(\min =12, \max =2,924)$. Trajectory $\mathrm{B}(\mathrm{n}=30)$ had a median cumulative dose of $2,077 \mathrm{mg}$ $(\min =975, \max =4,360)$. Trajectory $\mathrm{C}(\mathrm{n}=19)$ had a median cumulative dose of 4,020 $\mathrm{mg}$ $(\min =2,889, \max =5,543)$, and trajectory $\mathrm{D}(\mathrm{n}=6)$ had a median cumulative dose of 10,392 mg $(\min =8,978, \max =16,974)$. 'Pre-pregnancy only' $(\mathrm{n}=24)$ (not included in Figure 1) consisted of the gestations that had no antidepressant prescriptions between LMP and 32 weeks of gestation.

B. Average daily dose pregnancy trajectory-Three trajectories emerged when assessing average daily fluoxetine equivalent dose per week (Figure 3, top panel) in pregnancy. These trajectories represent a group that generally maintained a low average 
daily dose ( $<20 \mathrm{mg}$ ) for at least the first half of pregnancy (trajectory A, n=107), a group that tended to reduce or discontinue antidepressants in the first trimester (trajectory B, $n=29$ ) and a small group that maintained an average fluoxetine equivalent dose of approximately 40-60 mg/day throughout the pregnancy exposure period (trajectory C, $\mathrm{n}=6$ ). Similar to the cumulative trajectories, those that discontinued antidepressants prior to LMP $(n=24)$ were not included in Figure 3 but are referred to as 'Pre-pregnancy only' in analyses.

\section{Postnatal period}

Only 51 women (30.7\%) were prescribed an antidepressant in the first 3 months after delivery. This included 49 women with an antidepressant prescription between LMP and 32 weeks of gestation (34.5\%) and 2 women (8.3\%) with an antidepressant prescription in the 3 months prior to LMP who discontinued antidepressants between LMP and 32 weeks of gestation. Women in the highest cumulative dose trajectory in pregnancy (D) were most likely to remain on antidepressants in the postnatal period $(n=5 / 6,83.3 \%)$.

A. Cumulative postnatal trajectory-Postnatal cumulative antidepressant use was best described by 4 trajectory groups (Figure 2, bottom panel). Trajectory A ( $\mathrm{n}=120)$ had a median cumulative dose over the 13 weeks of $0 \mathrm{mg}(\min =0, \max =462)$. Trajectory $B(n=24)$ had a median cumulative dose of $756 \mathrm{mg}(\min =420, \max =1,356)$. Trajectory $\mathrm{C}(\mathrm{n}=14)$ had a median cumulative dose of $1,697 \mathrm{mg}(\min =1,300, \max =3,680)$, and trajectory $\mathrm{D}(\mathrm{n}=8)$ had a median cumulative dose of $4,333 \mathrm{mg}(\min =2,680, \max =6,400)$.

B. Average daily dose postnatal trajectory-Three trajectories emerged when analyzing average daily dose per week in the postnatal period (Figure 3, bottom panel). These were best characterized as a group with no or very low dose (generally $<10 \mathrm{mg}$ ) antidepressant prescriptions ( $\mathrm{n}=123$, trajectory $\mathrm{A}$ ), a group that tended to discontinue as the period progressed ( $n=35$, trajectory $B$ ) and a small group $(n=8$, trajectory $C$ ) with higher doses (generally $>40 \mathrm{mg}$ ) or doses that were maintained throughout the 3 months postpartum.

Fishers exact tests revealed that trajectory group membership in pregnancy was a good predictor of trajectory group membership in the postnatal period for both cumulative exposure trajectories $(\mathrm{p}=0.0004)$ and average daily dose trajectories $(\mathrm{p}=0.0002)$.

\section{Maternal characteristics and trajectory group}

EPDS score prior to 32 weeks differed by pregnancy trajectory group membership for both cumulative dose $(\mathrm{p}=0.04)$ and average daily dose per week $(\mathrm{p}=0.006)$. In group comparisons, individuals in trajectory A had higher EPDS scores by approximately 3.7 points than those that discontinued antidepressants prior to LMP. No other maternal characteristics statistically differed across cumulative or average daily pregnancy trajectories.

\section{Pregnancy trajectories and birth weight and gestational age at delivery}

In multivariable linear regression, the highest trajectory group (Trajectory $\mathrm{C}, \mathrm{n}=6$ ) in fluoxetine equivalent average daily dose was associated with an average reduced birth weight of 314.1 grams compared to the lowest use trajectory group (Trajectory A) (Table 2). This finding was similar by cumulative trajectory groups (Trajectory C-D vs. A), but 
confidence intervals crossed the null. Gestational age at delivery was not associated with any of the pregnancy trajectory groups. In sensitivity analyses, excluding twin gestations did not alter the findings (data not shown).

\section{Discussion}

A vast body of research has produced conflicting results on the risk of antidepressant exposure on pregnancy and birth outcomes (Way 2007; Alwan and Friedman 2009; Udechuku et al. 2010). Estimating the risk of antidepressant exposure to the developing fetus is greatly important, as untreated prenatal depression also carries its own risk profile to the fetus and the mother (Davalos et al. 2012). It is therefore imperative that we continue to evolve our methodology when modeling complex antidepressant exposures in pregnancy to provide the most accurate risk information to pregnant women and clinicians. In the present analysis, we used k-means longitudinal modeling to describe trajectories of antidepressant use in the pregnancy and postnatal periods.

To our knowledge, this is the first study that has utilized trajectory methods to describe antidepressant use during pregnancy. Although other studies have described antidepressant use according to the timing of use in pregnancy (Hayes et al. 2012) or the cumulative number of days on antidepressants (Oberlander et al. 2008), modeling trajectories of use reflects timing of initiation and discontinuation, average dose, cumulative dose, and changes in dose. By modeling trajectories, we may group women with similar profiles, allowing for a more robust analysis that reflects more of the complexity in exposure. In our analysis, four trajectories emerged when assessing the cumulative dose, and three trajectories emerged when assessing the average daily dose per week in pregnancy. In multivariable analysis, we identified an association between antidepressant use in the first 32 weeks of pregnancy and a modest reduction in birth weight (314 grams) among gestations with the greatest average daily dose compared with the lowest trajectory group. Although we adjusted for EPDS score in these models, we cannot know if residual confounding by indication or other unmeasured confounders biased these results. Additionally, we had very small strata in some of the trajectory groups which may result in unstable estimates. However, our findings are consistent in direction and magnitude with previous reports of an association between antidepressants and reduced birth weight (Oberlander et al. 2008; Udechuku et al. 2010; Nezvalová-Henriksen et al. 2016).

Employing trajectory methodology to this data revealed other interesting findings. Similar to previous reports, we did observe a distinct subgroup of women who discontinued antidepressant therapy around the time of pregnancy recognition (Figure 3, trajectory B) (Petersen et al. 2011). We anticipated that this group may have an increased risk for adverse birth outcomes due to a worsening of depression symptoms in the absence of pharmacotherapy; however, this was not observed in multivariable analysis. We are unable to determine if a non-pharmacologic therapy was substituted. We observed similar results in the group of women who discontinued antidepressants prior to LMP and did not resume use in the first 32 weeks of pregnancy. We did not find evidence of an increased risk of reduced birth weight or gestational age at delivery, and only 2 women (8\%) resumed antidepressants in the early postnatal period. 
When interpreting these results, it is important to be aware of the limitations. Our data originated from medical records; though the clinics had implemented medication reconciliation review for each patient visit during the study period, we do not know for certain if these prescriptions were filled or taken and we can only estimate the timing of antidepressant discontinuation. Although we attempted to be conservative in our estimates of the date of discontinuation, we may still have overestimated durations of use if women did not fill or take the medication as prescribed. We would anticipate any bias to be nondifferential to trajectory assignment, potentially attenuating results. Also, $58(35 \%)$ of women did not have evidence of a visit with a primary care, internal medicine, or OBGYN provider within UC San Diego Health in the 365 days prior to LMP-3 months. We cannot be sure that we captured all antidepressant use prescribed to this group in early pregnancy, as it may have been prescribed elsewhere. However, these women did not differ with respect to trajectory group assignment. Also, our relatively small sample resulted in small trajectory groups and precluded our ability to investigate dichotomous outcomes such as low birthweight or preterm birth; larger data sets are necessary for these and other outcomes of interest. Finally, this study used data from a single medical center, and the trajectory patterns identified may not be generalizable to other populations.

Analyzing medication use in pregnancy with longitudinal trajectory methods may not be appropriate for all outcomes. When studying outcomes with narrow etiologically relevant timeframes, such as many birth defects, highest observed daily dose or total cumulative dose or days of exposure during the sensitive window may be more useful than trajectory methods. However, for other outcomes with unknown etiologies, such as preterm birth, offspring birth weight or neurodevelopmental outcomes, these trajectories hold promise. Future directions include using 3-d modeling (kml3d) (Genolini et al. 2015) to test interactions between medications.

In summary, we employed k-means longitudinal trajectory modeling to antidepressant use in pregnancy and found the highest trajectory of exposure to be associated with a modest reduction in birth weight. These profiles offer a more meaningful way of understanding and categorizing medication exposures in pregnancy.

\section{Supplementary Material}

Refer to Web version on PubMed Central for supplementary material.

\section{Acknowledgments}

Gretchen Bandoli is supported by the National Institutes of Health, Grant TL1TR001443. Kristin Palmsten is supported by a career development award from the Eunice Kennedy Shriver National Institute of Child Health \& Human Development, National Institutes of Health (K99HD082412). The content is solely the responsibility of the authors and does not necessarily represent the official views of the NIH. The other authors have no funding sources for this work to declare.

\section{References}

Alwan S, Friedman JM. Safety of Selective Serotonin Reuptake Inhibitors in Pregnancy. CNS Drugs. 2009; 23:493-509. DOI: 10.2165/00023210-200923060-00004 [PubMed: 19480468] 
Andrade SE, Raebel MA, Brown J, et al. Use of antidepressant medications during pregnancy: a multisite study. Am J Obstet Gynecol. 2008; doi: 10.1016/j.ajog.2007.07.036

Caliniski T, Harabasz J. A Dendrite Method for Cluster Analysis. Commun Stat Theory Methods. 1974; 3:1-27.

Cooper WO, Willy ME, Pont SJ, Ray WA. Increasing use of antidepressants in pregnancy. Am J Obstet Gynecol. 2007; 196:1-5. DOI: 10.1016/j.ajog.2007.01.033 [PubMed: 17240216]

Cox JL, Holden JM, Sagovsky R. Detection of postnatal depression. Development of the 10-item Edinburgh Postnatal Depression Scale. Br J Psychiatry. 1987; 150:782-786. DOI: 10.1192/bjp. 150.6.782 [PubMed: 3651732]

Davalos DB, Yadon CA, Tregellas HC. Untreated prenatal maternal depression and the potential risks to offspring: A review. Arch Womens Ment Health. 2012; 15:1-14. DOI: 10.1007/ s00737-011-0251-1 [PubMed: 22215285]

Davies D, Bouldin D. A Cluster Separation Measure. IEEE Trans Pattern Anal Mach Intell. 1979; 1:224-227. [PubMed: 21868852]

Franklin JM, Shrank WH, Pakes J, et al. Group-based Trajectory Models. Med Care. 2013; 51:789796. DOI: 10.1097/MLR.0b013e3182984c1f [PubMed: 23685406]

Genolini C, Alacoque X, Sentenac M, Arnaud C. kml and kml3d: R Packages to Cluster Longitudinal Data. J Stat Softw. 2015; 65:1-34. DOI: 10.1359/JBMR.0301229

Genolini C, Falissard B. Package "kml”. 2016:1-33.

Genolini C, Falissard B. Kml: A package to cluster longitudinal data. Comput Methods Programs Biomed. 2011; 104:e112-e121. DOI: 10.1016/j.cmpb.2011.05.008 [PubMed: 21708413]

Hayasaka Y, Purgato M, Magni LR, et al. Dose equivalents of antidepressants: Evidence-based recommendations from randomized controlled trials. J Affect Disord. 2015; 180:179-184. doi: http://dx.doi.org/10.1016/j.jad.2015.03.021. [PubMed: 25911132]

Hayes RM, Wu P, Shelton RC, et al. Maternal antidepressant use and adverse outcomes: a cohort study of 228,876 pregnancies. Am J Obstet Gynecol. 2012; 207:49.e1-49.e9. DOI: 10.1016/j.ajog. 2012.04.028 [PubMed: 22727349]

Hurault-Delarue C, Chouquet C, Savy N, et al. How to take into account exposure to drugs over time in pharmacoepidemiology studies of pregnant women? Pharmacoepidemiol Drug Saf. 2016; 25:770-777. DOI: 10.1002/pds.4000 [PubMed: 27018245]

Hurault-Delarue C, Chouquet C, Savy N, et al. Interest of the trajectory method for the evaluation of outcomes after in utero drug exposure: example of anxiolytics and hypnotics. Pharmacoepidemiol Drug Saf. 2017; 26:561-569. DOI: 10.1002/pds.4199 [PubMed: 28337823]

Huybrechts KF, Palmsten K, Mogun H, et al. National trends in antidepressant medication treatment among publicly insured pregnant women. Gen Hosp Psychiatry. 2013; 35:265-271. DOI: 10.1016/ j.genhosppsych.2012.12.010 [PubMed: 23374897]

Jacobs P, Ang A, Hillhouse MP, et al. Treatment outcomes in opioid dependent patients with different buprenorphine/naloxone induction dosing patterns and trajectories. Am J Addict. 2015; 24:667675. DOI: 10.1111/ajad.12288 [PubMed: 26400835]

Nezvalová-Henriksen K, Spigset O, Brandlistuen RE, et al. Effect of prenatal selective serotonin reuptake inhibitor (SSRI) exposure on birthweight and gestational age: a sibling-controlled cohort study. Int J Epidemiol. 2016; 45:2018-2029. [PubMed: 27188860]

Oberlander TF, Warburton W, Misri S, et al. Effects of timing and duration of gestational exposure to serotonin reuptake inhibitor antidepressants: Population-based study. Br J Psychiatry. 2008; 192:338-343. DOI: 10.1192/bjp.bp.107.037101 [PubMed: 18450656]

Palmsten, K., Rolland, M., Hebert, M., et al. Patterns of Prednisone Use during Pregnancy: Daily and Cumulative Dose [abstract]. Health Care Systems Research Network; San Diego, CA: 2017.

Petersen I, Gilbert RE, Evans SJW, et al. Pregnancy as a major determinant for discontinuation of antidepressants: An analysis of data from The Health Improvement Network. J Clin Psychiatry. 2011; 72:979-985. DOI: 10.4088/JCP.10m06090blu [PubMed: 21457681]

Ray S, Stowe ZN. The use of antidepressant medication in pregnancy. Best Pract Res Clin Obstet Gynaecol. 2014; 28:71-83. DOI: 10.1016/j.bpobgyn.2013.09.005 [PubMed: 24211026] 
Ray, S., Turi, RH. Determination of Number of Clusters in K-Means Clustering and Application in Colour Image Segmentation. Proc 4th Int Conf Adv Pattern Recognit Digit Tech; 1999. p. 137-143.

Riegel B, Lee CS, Ratcliffe SJ, et al. Predictors of objectively measured medication nonadherence in adults with heart failure. Circ Hear Fail. 2012; 5:430-436. DOI: 10.1161/CIRCHEARTFAILURE. 111.965152

Siu AL. (USPSTF) and the USPSTF. Screening for depression in adults: Us preventive services task force recommendation statement. JAMA. 2016; 315:380-387. [PubMed: 26813211]

Udechuku A, Nguyen T, Hill R, Szego K. Antidepressants in Pregnancy: a Systematic Review. Aust New Zeal J Psychiatry. 2010; 44:978-996. DOI: 10.3109/00048674.2010.507543

Way CM. Safety of Newer Antidepressants in Pregnancy. Pharmacother J Hum Pharmacol Drug Ther. 2007; 27:546-552. DOI: 10.1592/phco.27.4.546 


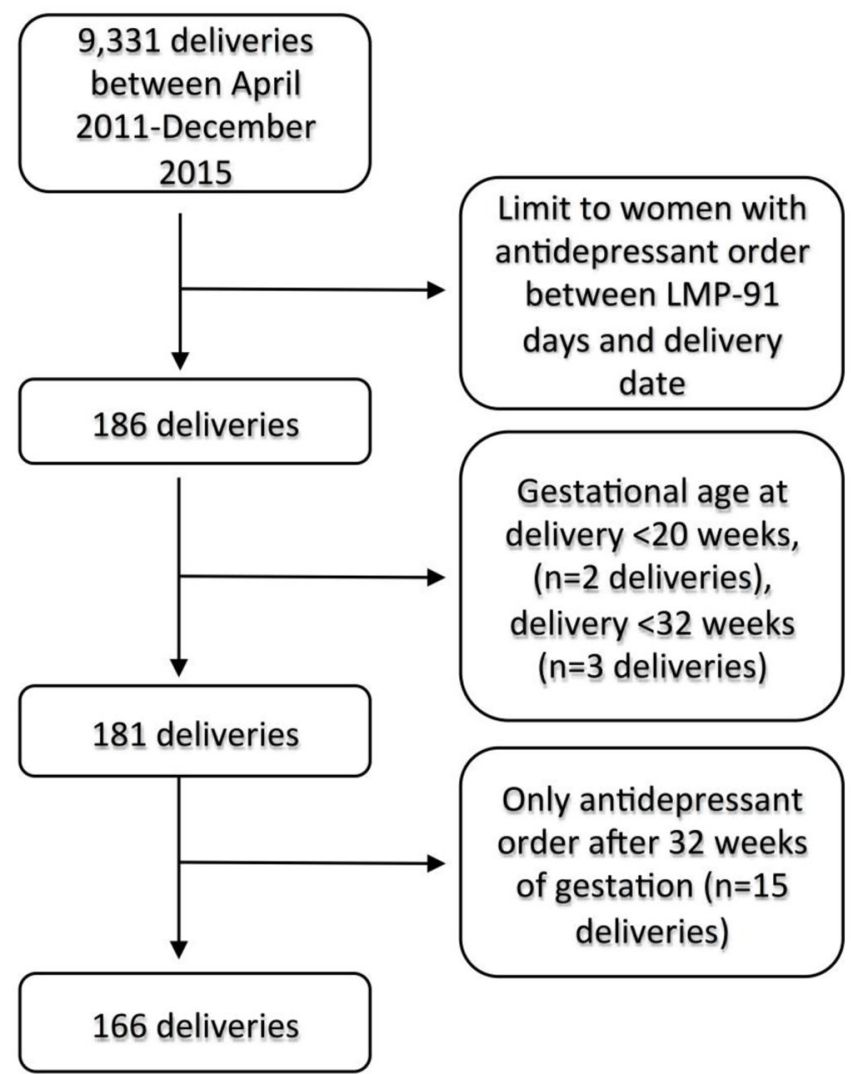

Figure 1.

Flow chart for inclusion in the study cohort 

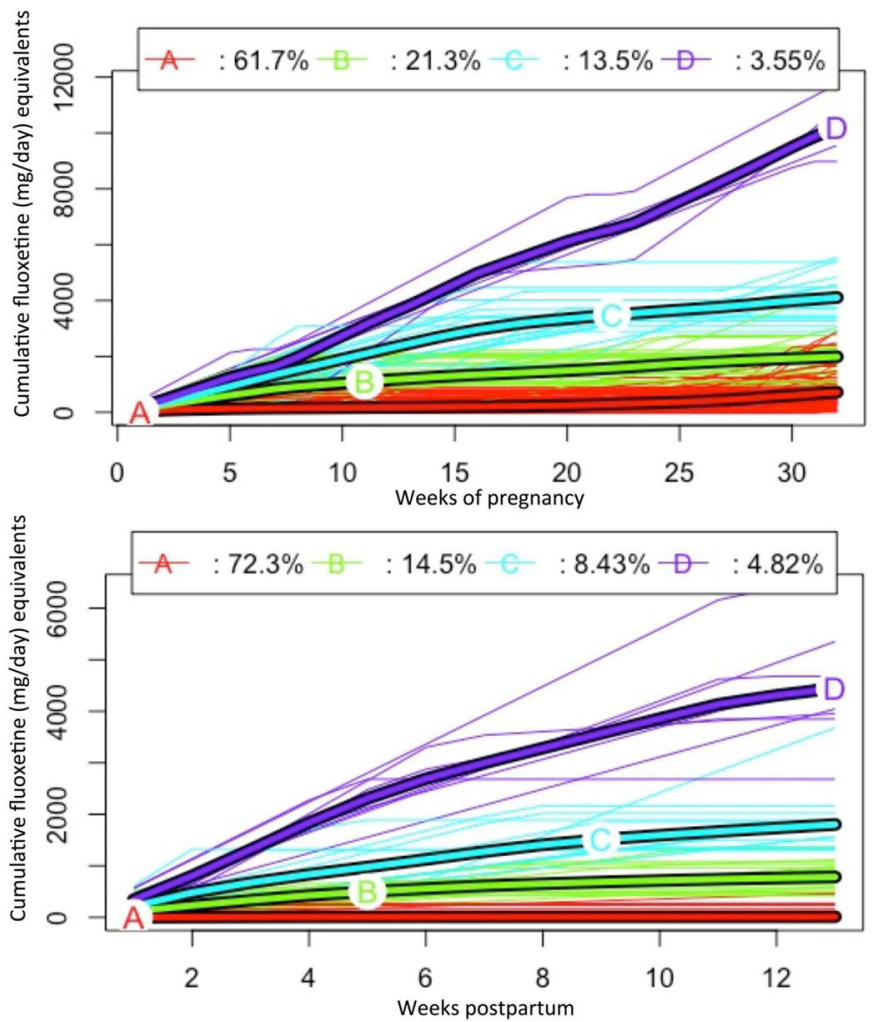

Figure 2.

Cumulative fluoxetine equivalents $(\mathrm{mg})$ per week from LMP to 32 weeks of gestation. Individuals with no antidepressant prescriptions between LMP and 32 weeks of gestation $(n=24)$ are excluded from trajectories. One outlier with disproportionally high cumulative dose in pregnancy was removed; $\mathrm{n}=141$ (top panel). Cumulative fluoxetine equivalents (mg) per week from delivery date through 13 weeks postpartum; n=166 (bottom panel) 

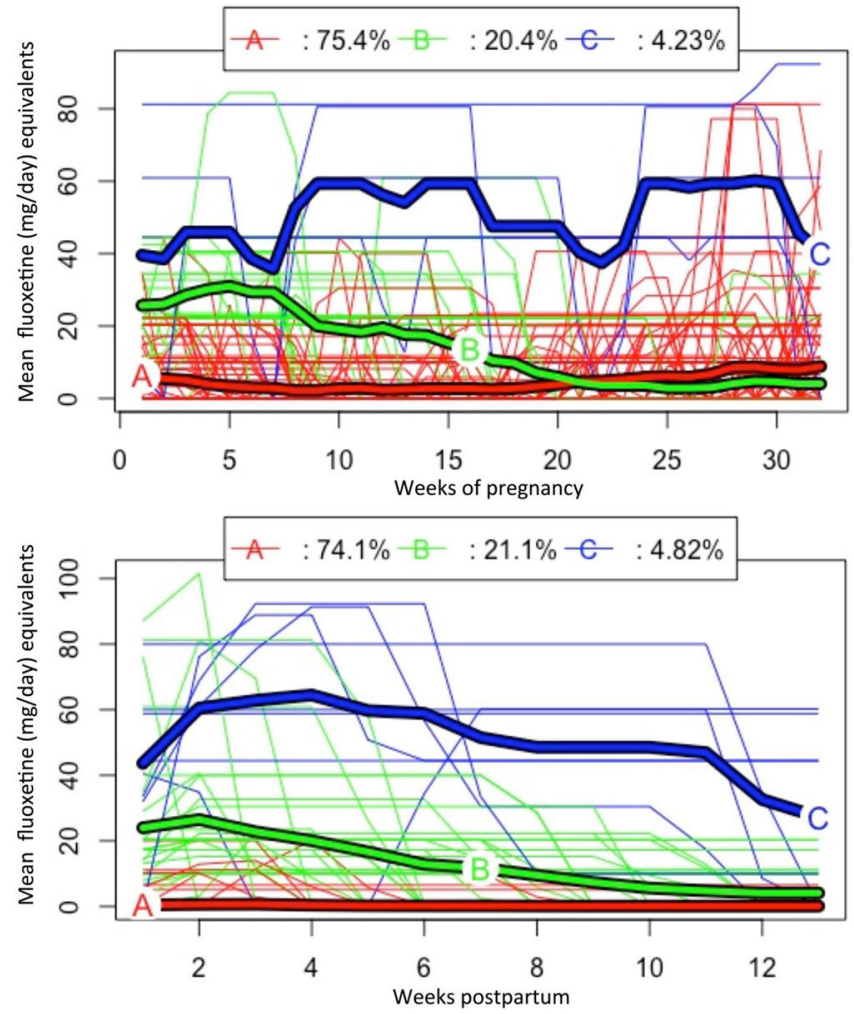

Figure 3.

Average daily fluoxetine equivalents $(\mathrm{mg})$ per week from LMP to 32 weeks of gestation. Individuals with no antidepressant prescriptions between LMP and 32 weeks of gestation $(n=24)$ are excluded from trajectories; $n=142$ (top panel). Average daily fluoxetine equivalents (mg) per week from delivery date through 13 weeks postpartum; n=166 (bottom panel) 
Bandoli et al.

을

要

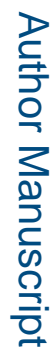

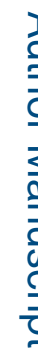

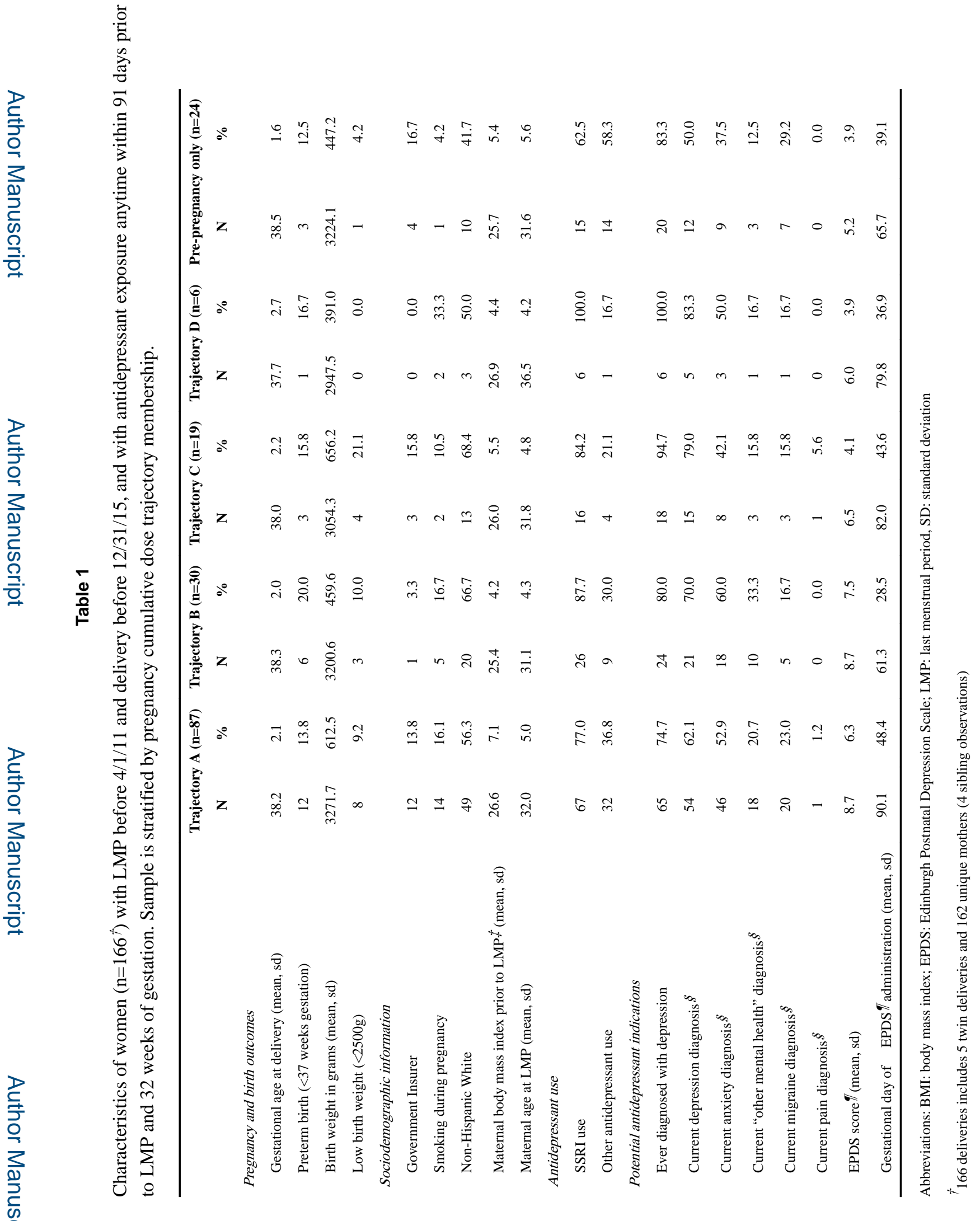

Arch Womens Ment Health. Author manuscript; available in PMC 2019 August 01. 

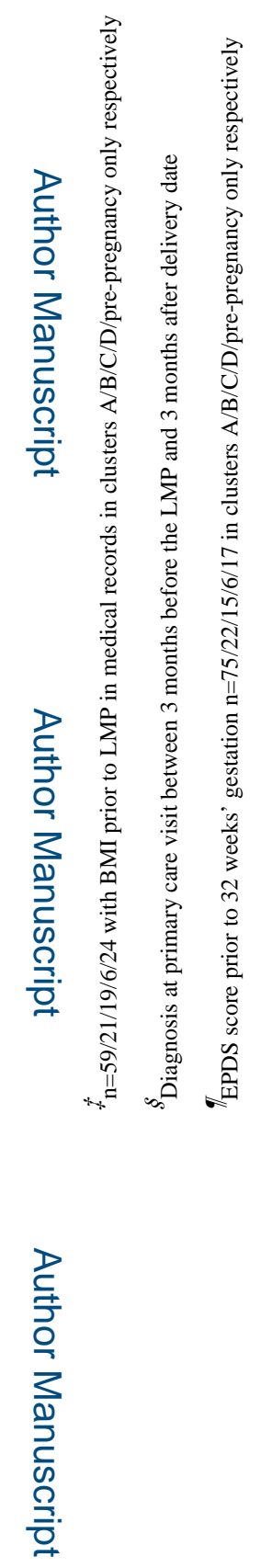

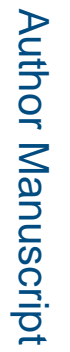

Arch Womens Ment Health. Author manuscript; available in PMC 2019 August 01. 


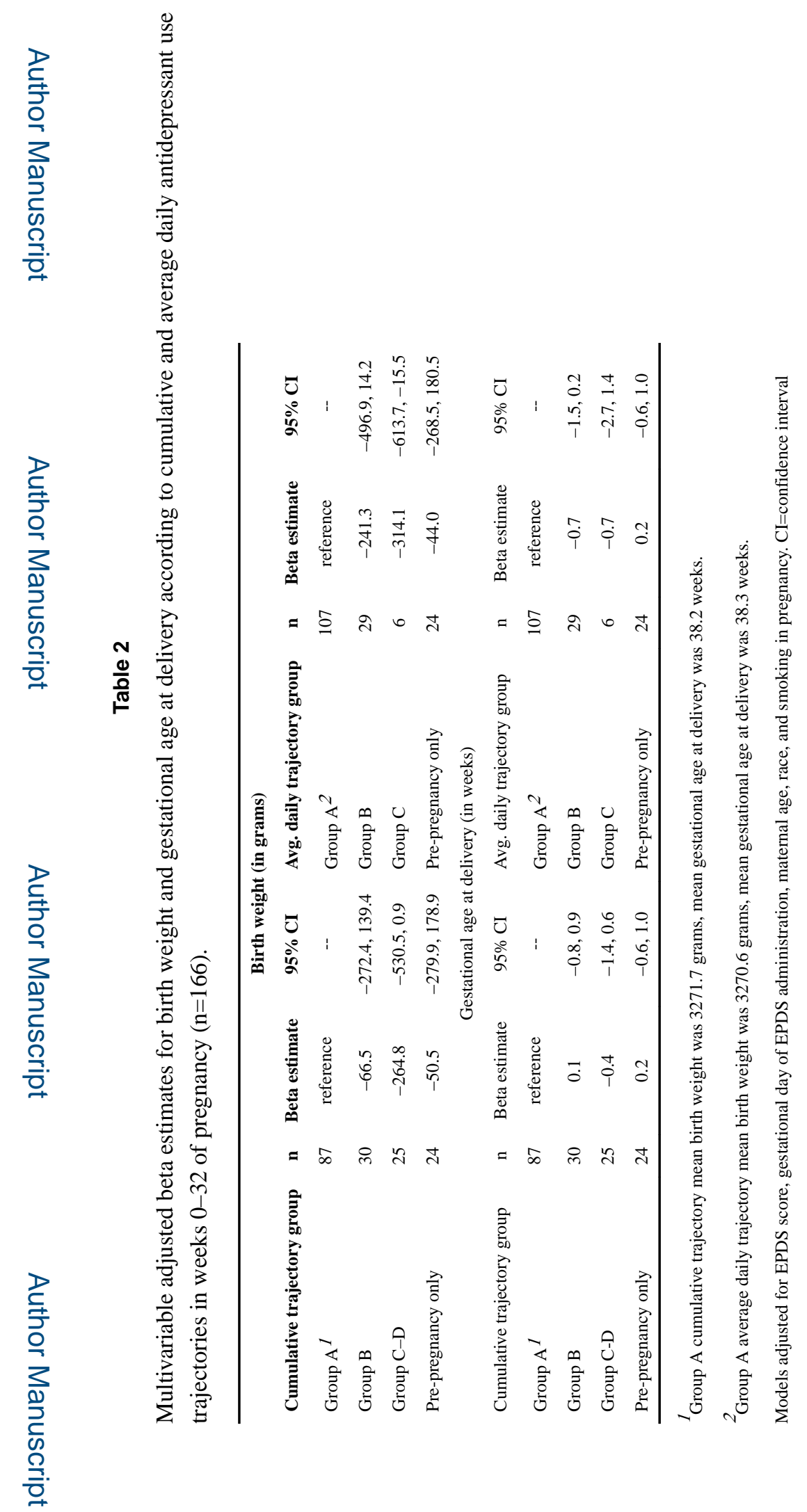

Arch Womens Ment Health. Author manuscript; available in PMC 2019 August 01. 